\title{
Política petrolera venezolana en tiempos de catástrofe
}

\section{Carlos Mendoza Pottellá ${ }^{I}$}

\section{"Venezuela se acerca a la debacle" II}

Resumo: A crise política, social e econômica que a Venezuela enfrenta hoje é o resultado da ação de uma série de fatores políticos, geopolíticos e econômicos, que influenciam e se manifestam de forma decisiva na saúde e no futuro de sua indústria petrolífera. que conseguiu gerar mais de $90 \%$ de sua renda externa e que, por esse motivo, mantém por nove décadas uma sociedade viciada em alavancagem externa e incapaz de desenvolver uma economia produtiva autônoma. Este trabalho enfoca a análise dos determinantes da involução da indústria do petróleo, alguns dos quais têm seus determinantes externos, no cenário geopolítico do século passado e presente, mas outros, a meu ver, fundamentais, que têm É o produto de políticas econômicas e de gestão, específicas para esse setor industrial. Em particular, trato das críticas ao planejamento petrolífero venezuelano que começa a ser elaborado após a "reversão" à Nação, em 1976, das concessões concedidas a empresas internacionais pelo desenvolvimento da atividade petrolífera desde 1908. Um planejamento de mundos ilusórios, baseado em uma percepção errônea das circunstâncias naturais que determinaram o maior acúmulo de petróleo extra pesado no mundo em nosso país. Um planejamento no qual eles continuam a influenciar, mesmo a partir dos postos de comando da indústria "nacionalizada", os interesses do capital internacional do petróleo e suas respectivas metrópoles.

Palavras-chave: Economia, geopolítica, petróleo, planejamento, políticas públicas.

\section{Venezuelan oil policy in catastrophic times}

Abstract: The political, social and economic crisis facing Venezuela today is the result of the action of a series of political, geopolitical and economic factors, all of which influence and manifest themselves in a decisive way in the health and future of its oil industry, activity that it has managed to generate more than 90 percent of its external income and that, for this reason, it has sustained for nine decades a society addicted to external leverage and unable to develop an autonomous productive economy. This work focuses on the analysis of the determinants of the involution of the oil industry, some of which have their external determinants, in the geopolitical scenario of the past and present century, but others, in my way of seeing fundamental, that have It has been the product of economic and management policies, specific to that industrial sector. In particular, I address the criticism of Venezuelan oil planning that begins to be designed after the "reversion" to the Nation, in 1976, of the concessions granted to international corporations for the development of oil activity since 1908. illusory worlds, based on a misperception of the natural circumstances that determined the largest accumulation of extra heavy oil in the world in our country. A planning in which they continue to influence, even from the command posts of the "nationalized" industry, the interests of international oil capital and their respective metropolis.

Keywords: Economy, geopolitics, oil, planning, public policies. 


\section{POLÍTICA PETROLERA VENEZOLANA EN TIEMPOS DE CATÁSTROFE}

\section{CARLOS MENDOZA POTTELLÁ}

Artigo recebido em 15/10/2019 e aprovado em 27/11/2019.

\section{Introducción}

Después de haber generado durante más de cien años una gigantesca renta, producto de la fertilidad petrolera de su tierra y el carácter de necesidad estratégica y económica universal que adquirió este recurso bajo el control de las grandes potencias y sus monopolios, luego de haber intentado infructuosamente durante todo ese lapso de "sembrar" unos ingresos, que se suponían extinguibles por su carácter minero, para que fructificaran en una economía autónoma y autosustentable, venciendo el "Efecto Venezuela" III, el país se encuentra hoy viviendo una aguda y peligrosa crisis en todos los órdenes socioeconómicos y políticos.

La expresión más más evidente de este drama se encuentra a en el derrumbe, entre 2008 y 2019, de la producción petrolera en más de 2,5 millones de barriles diarios, como lo expresan las cifras que generan el siguiente gráfico. ${ }^{\text {IV }}$

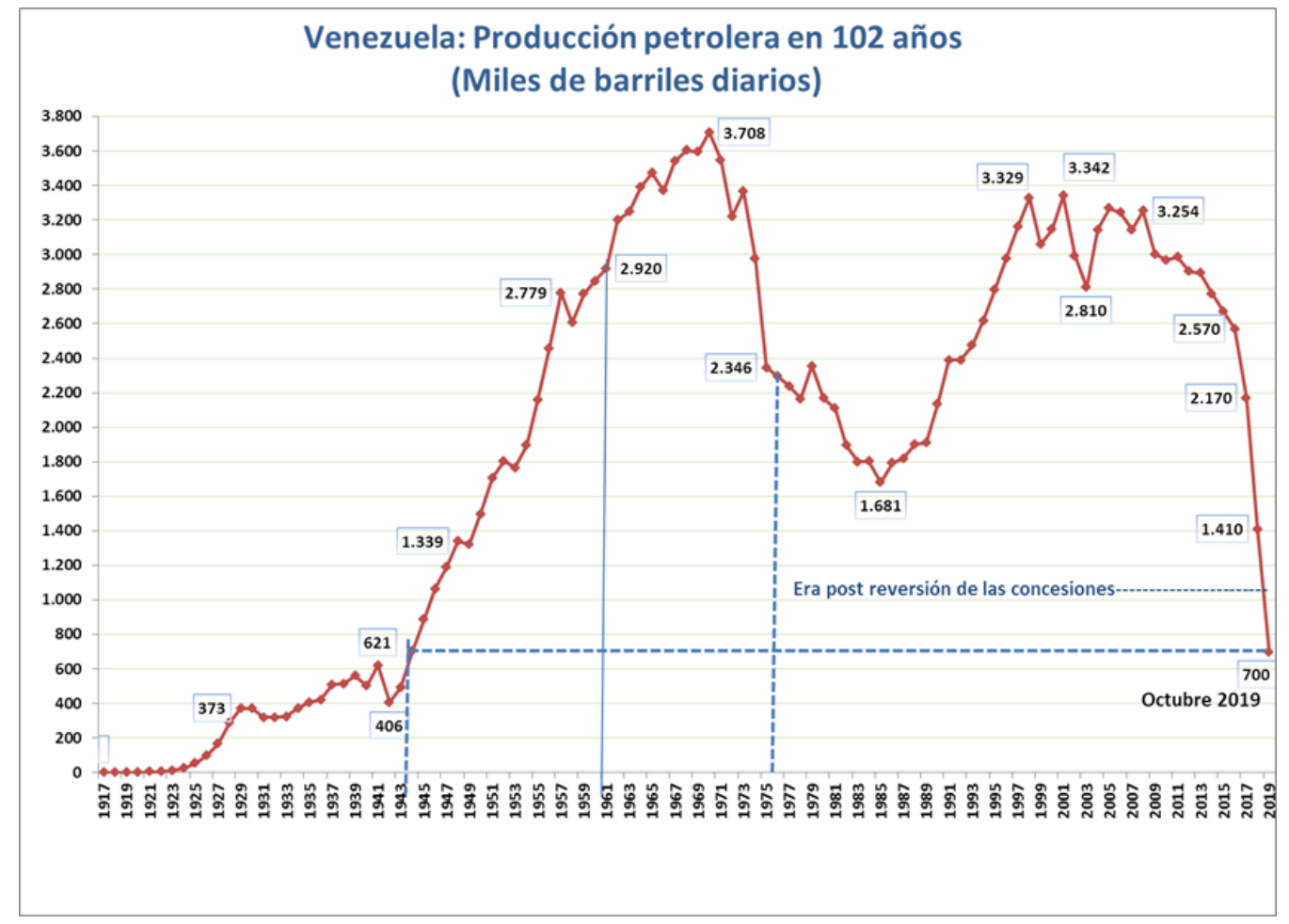

El factor fundamental en la gestación de este descalabro se encuentra en el inexorable agotamiento físico de sus recursos petroleros convencionales $y$, concurrentemente, de su capacidad generadora de excedentes, circunstancias éstas empeoradas por una ruinosa planificación de mundos ilusorios, fincada en la existencia física, bajo el subsuelo del norte del Orinoco, de un océano petrolero: “...la mayor acumulación de petróleo extrapesado está en la faja de petróleo pesado del Orinoco venezolano, que contiene el 90 por ciento del petróleo extrapesado del mundo cuando se mide in situ.". $\mathrm{V}$ 


\section{POLÍTICA PETROLERA VENEZOLANA EN TIEMPOS DE CATÁSTROFE}

\section{CARLOS MENDOZA POTTELLÁ}

La discusión de este y otros aspectos de la dramática involución contemporánea de la industria petrolera venezolana la he expuesto en recientes trabajos publicados en el blog https://petroleovenezolano.blogspot.com y otros portales electrónicos, de manera particular en el titulado “Recursos, Reservas, Faja y Lutitas” VI .

El presente trabajo está dedicado a una revisión más específica de ese proceso: el análisis de una de las circunstancias causales del mismo, a la cual considero uno de los factores generadores de todas las demás: Justamente, las fantasías inducidas sobre la posesión de las mayores reservas petroleras del mundo y los fiascos ruinosos que las mismas han generado... y amenazan con seguirlo haciendo.

En los años 70 del Siglo XX, en medio de las convulsiones de la Guerra ÁrabeIsraelí (del Yom Kippur o del Ramadán) de 1973 y el subsiguiente embargo petrolero árabe, tomaron estado público algunas predicciones sombrías sobre la "crisis energética" y el "pico del petróleo", que para los yacimientos convencionales norteamericanos ocurriría en 1971, año a partir del cual se iniciaría el inevitable descenso de su reservas y capacidades de producción, tal como lo estimara certeramente, en 1956, el Geólogo Marion King Hubbert.

Para esa misma época, las también antiguas predicciones del futurólogo y estratega militar Herman Khan, de la RAND Corporation, cuyos "escenarios" colocaban a 2050 como la fecha en que se iniciaría el agotamiento generalizado del petróleo, comenzaron a acercarse peligrosamente hacia los años finales del pasado siglo, dado el crecimiento exponencial del consumo mundial de petróleo entre $1946 \mathrm{y}$ 1972.

Con esas perspectivas se acentuaron las preocupaciones por la seguridad energética de los Estados Unidos y demás países aliados y se instaura la Agencia Internacional de Energía como un centro coordinador de políticas de los países de la OCDE, se promovieron iniciativas de ahorro energético y sustitución de petróleo por otras fuentes energéticas y de petróleo OPEP por el de cualquier otra procedencia. Todo ello en un ambiente de agudización de la Guerra Fría.

Un ejemplo de la crispación de esos años fue el discurso del "Estado de la Unión" de Jimmy Carter en 1979, en el cual expuso un severo programa de seguridad energética, para enfrentar tendencias que de no ser contenidas determinarían que: "para mantener los ritmos del consumo global de petróleo se requerirá descubrir cada 9 meses una nueva Alaska, cada año una nueva Texas y cada tres años una nueva Arabia Saudita". VII

Desde los inicios de esa "crisis", se planteaba que al acabarse el petróleo convencional quedarían sólo dos grandes acumulaciones petroleras: la Faja del Orinoco y las Arenas Bituminosas de Canadá. Otras, más pequeñas quedaban fuera del alcance occidental, en la Unión Soviética. Esas circunstancias eran evaluadas con pesimismo respecto a Venezuela en estudios realizados para el Congreso estadounidense, debido a la preeminencia que tenía en este país el discurso conservacionista del Dr. Juan Pablo Pérez Alfonzo, ministro fundador de la OPEP en 1960 y para entonces máxima referencia nacional en materia de política petrolera.

Circuló entonces en los medios políticos venezolanos una versión, no confirmada, pero dada por verídica, de que la dictadura brasileña de entonces, la cual se promocionaba a sí misma como el "sub-imperialismo", había establecido, en su "Projeto Calha Norte" de los años 70, que un servicio que ella podría prestar a la 


\section{POLÍTICA PETROLERA VENEZOLANA EN TIEMPOS DE CATÁSTROFE}

\section{CARLOS MENDOZA POTTELLÁ}

seguridad energética de "Occidente" era tomar todo el sureste de Venezuela para garantizar la explotación de la Faja.

La alarma en los círculos oficiales venezolanos fue cónsona con la magnitud de la supuesta amenaza: El Presidente Rafael Caldera recibió la visita de James Akins, Zar energético de Richard Nixon, ante el cual dio seguridades de que los venezolanos si estábamos dispuestos a explotar la Faja y que no era necesaria la intervención brasileña.

Como muestra, se anunció oficialmente el cambio del nombre tradicional de Faja Bituminosa del Orinoco por el de Faja Petrolífera del Orinoco. Desde luego, Pérez Alfonzo protestó, afirmando que eso era poner sobre el mostrador lo que se guardaba para las futuras generaciones.

El proceso geopolítico global continuó su desarrollo y una de las movidas estratégicas de los planificadores energéticos de las grandes corporaciones fue el ofrecimiento, hecho a finales de 1972 por el enclave coordinador de las mismas, a los países ribereños del Golfo Arábico-Pérsico, de una nacionalización parcial, escalonada, cuya puesta en práctica por algunos de ellos en los años siguientes, determinó la aparición en los mercados mundiales del llamado "crudo de participación", rara avis no sujeta a las disposiciones monopólicas imperantes absolutamente hasta entonces. Esa política fue descrita por el propio James Akins VIII como producto de la necesidad de destapar la olla a punto de explotar del nacionalismo árabe.

Por su parte, en Venezuela había comenzado a regir, en 1971 y por virtud de una creciente conciencia reivindicativa de la participación nacional en los ingresos de la industria establecida sobre sus recursos petroleros, "Ley Sobre Bienes Afectos a Reversión", en la cual se normaban los mecanismos y recursos financieros que garantizaban la intangibilidad operativa de los yacimientos y equipos de la industria petrolera, hasta las fechas prevista para el inicio de la reversión de las concesiones, a partir de 1983, cuando las mismas vencían en un $80 \%$. ${ }^{\text {IX }}$

Sin embargo, la estrategia de las corporaciones no permitiría esa espera, onerosa de por sí para ellas, dado el costo de cumplir con las obligaciones establecidas en la referida Ley. Un costo creciente después de 50 años de explotación intensiva, como se constatará posteriormente en las cuentas de la industria "nacionalizada" después de 1976.

Fue así entonces, como entre 1972 y 1973 los Presidentes de las mayores corporaciones concesionarias, Shell de Venezuela y de Creole Petroleum Corporation (J.J. de Liefde y Robert N. Dolph), expresaron claramente la disposición de sus respectivas empresas a seguir colaborando con el país después de la reversión de las concesiones. ${ }^{X}$

Ello desató una euforia nacionalista entre los venezolanos, evidente en las múltiples declaraciones posteriores registradas en la obra antes citada. En ese ambiente político se produjo el "adelanto" a 1976, de la reversión prevista para 1983. ${ }^{\mathrm{XI}}$

En verdad, se trataba de la puesta en práctica de una fórmula precautelativa del capital internacional, ensayada desde los años 60 con el proceso de “venezolanización”, de la gerencia y ejecutada con precisión en los 70. En agosto de 1975, cuatro meses antes del decreto supuestamente nacionalizador: Creole Petroleum, la filial de Exxon, creó de su seno a Lagoven, la operadora nacionalizada que la 


\section{POLÍTICA PETROLERA VENEZOLANA EN TIEMPOS DE CATÁSTROFE}

\section{CARLOS MENDOZA POTTELLÁ}

sustituiría, dotada de una Junta Directiva constituida por los "nativos" que integraban la Junta de Creole. De igual manera, Shell creó a Maraven, Mobil creó a Llanoven, Gulf creó a Meneven, Texas creó a Deltavén etc., hasta 13 "operadoras". Es así como, el $1^{\circ}$ de enero de 1976, aparece como primer Presidente de Maraven, quien había sido hasta el día anterior el Presidente de la Compañía Shell de Venezuela, fórmula que se repite en distintos niveles en las otras operadoras.

\footnotetext{
El "adelanto de la reversión" que se produjo en 1975-76, sedicentemente denominado "nacionalización", no fue más que el resultado de una composición de fuerzas nacionales e internacionales que lograron, como siempre, convertir una consigna nacionalista en el mejor camino para fortalecer la presencia del gran capital transnacional en las fases más rentables del negocio petrolero venezolano. ${ }^{\mathrm{XI}}$
}

Ese fue el resultado de las negociaciones tras bastidores que, con el nombre de "avenimiento", dieron luz verde a ese adelanto y garantizaron a las corporaciones internacionales jugosas indemnizaciones sobre activos largamente depreciados, contratos de asistencia técnica y comercialización mediante los cuales se les remuneró con creces su tan reclamado, cuan inmerecido, lucro cesante y permanencia en todos los emprendimientos de sus antiguas filiales, ahora "operadoras" nacionalizadas. ${ }^{\text {XIII }}$

Ya en estos contratos de asistencia técnica y comercialización se inicia el proceso de desmontaje del aparato de control y fiscalización estructurado por el Estado venezolano a lo largo de décadas. En ellos se consagró, por primera vez, la renuncia a la soberanía impositiva, al establecer una fórmula automática para compensar todo intento de incremento de las tasas impositivas vigentes a la firma del contrato.

Igualmente, allí, por primera vez, se renunció a la "inmunidad de jurisdicción", al establecer, en contravención del Artículo 127 de la Constitución Nacional, el arbitraje internacional como medio para dirimir los desacuerdos entre las partes contratantes.

El poder petrolero "venezolanizado" tardó poco en retomar los intereses estratégicos corporativos de sus antiguos patrones sobre la Faja:

\footnotetext{
At present we are actively working on two specific projects in the Orinoco Oil Belt Area... (Se refiere al DSMA, Desarrollo del Sur de Monagas y Anzoátegui y al Guanipa 100+, n. n.)

The investment required for these two projects is estimated at eight billion dollars of 1979 and includes substantial expenditures for infraestructure as this is virtually virgin territory. Other projects of similar magnitude will necessary to raise production level from the entire Orinoco Oil Belt to one million barrels per day the year 2000, as contemplated in Venezuela's long range energy plans. ...

The total investment program that we have undertaken will require some $\$$ 25 billion over the next six years. Afterwards and until the end of this century, the yearly rate of investment is likely to average some $\$ 5$ billion (in 1980 dollars. We expect to generate most, if not all, of this capital internally, through reinvestment of future earn. Up to now, we have set aside more than six billion dollars to be used exclusively for future oil development activities." XIV
} 


\title{
POLÍTICA PETROLERA VENEZOLANA EN TIEMPOS DE CATÁSTROFE
}

\section{CARLOS MENDOZA POTTELLÁ}

El majestuoso plan del antiguo Vice-Presidente de la Creole Petroleum Corp., la mayor inversión en el mundo petrolero de esa época, 100.000 millones de dólares en 20 años, se basaba en una expectativa de precios crecientes hasta el año 2000, que suponía permanente la reciente evolución exponencial de los mismos, desde menos de 2 dólares en 1960 hasta 34 dólares en el año en que hablaba, 1981.

Según las proyecciones de la Dirección de Comercio y Suministro de PDVSA, el precio del crudo de $24^{\circ} \mathrm{API}$, promedio típico venezolano, que se cotizaba entonces en $32,53 \$ / b l$, alcanzaría en 2000 56,22\$/bl. En ese mismo lapso, el crudo pesado de $10^{\circ} \mathrm{API}$, tomado como referencia de los extrapesados que se producirían en la Faja, pasarían de los 21,40 \$/bl corrientes a 35,55 en 2000. De todo lo cual se infería que, en este último año, el precio de la mezcla $70 / 30$ sería de $44,89 \$ / b 1 .{ }^{X V}$

\footnotetext{
"En los estudios de exploración y caracterización realizados durante los años1979 al 1984 de 56.000 Kilómetros cuadrados que cubren la FPDO, donde se perforaron 669 pozos con longitud total de 643.000 metros, 5.500 kilómetros de registros eléctricos-petrofîsicos, 15.000 kilómetros de líneas sísmicas, el corte de 4500 metros de núcleos y 288 análisis de laboratorio (Análisis de Núcleos); A un costo de 650 millones de dólares USA"XVI
}

La realidad se presentó de inmediato, el ascenso de los precios, iniciado en el entorno de los $2 \$ /$ bl en 1972 , alcanzó los mencionados $34 \$ /$ bl de 1983 , pero inició allí mismo una caída que llevó los correspondientes al petróleo venezolano en 1998, a 5 dólares al barril, a precios de 1984. Ese primer sueño de la Faja, "el megadisparate de PDVSA" XVII quedaba así saldado, con pérdidas en el orden de los miles de millones de dólares.

A pesar de la caída de los precios del petróleo, la contumacia expansiva a cualquier costo no se detuvo, ensayando toda clase de negocios para eludir el compromiso nacional de defensa de los mismos, asumido en el seno de la OPEP y, por el contrario, promoviendo la salida del país de esa Organización: Los planificadores mayores de PDVSA proponían en 1994 un plan para duplicar la producción hacia el 2002. Consciente de la experiencia anterior, uno de ellos pontificaba: "compensaremos la caída de los precios con más producción".

El "escenario productor", tenía una precondición:

\begin{abstract}
"La alta tributación fiscal, la cual ni siquiera permite en el futuro inmediato hacer las inversiones necesarias para compensar la declinación y mantener la capacidad de producción, es el principal obstáculo que encuentra la Industria Petrolera Nacional para su desarrollo..."

"Por ello el Plan de la IPPCN ${ }^{\text {XVIII }}$ se basó en la premisa fundamental de aliviar la carga tributaria sobre PDVSA mediante reducción progresiva del valor fiscal de exportación hasta su total eliminación en tres o cuatro años..." "Cabe resaltar que un paso primordial en la realización de este plan lo ha constituido la reciente aprobación, por parte del Congreso Nacional, de la reducción gradual del Valor Fiscal de Exportación, que lo llevará de un 16\% en el presente año a un $8 \%$ en 1994 , a un $4 \%$ en 1995 y a su total eliminación en 1996". XIX
\end{abstract}

El éxito del escenario productor, enfrentado al rentismo parasitario fue realmente colosal: los ingresos petroleros del gobierno central cayeron de $83 \%$ en 1990 a $26 \%$ en 


\section{POLÍTICA PETROLERA VENEZOLANA EN TIEMPOS DE CATÁSTROFE}

\section{CARLOS MENDOZA POTTELLÁ}

1998. Al punto de que se planteó, por primera vez, la necesidad de exigirle a PDVSA la declaración de dividendos para rellenar el hueco fiscal que se asomaba.

La producción creció, pero junto con ella los costos, con lo cual, el resultado neto para la Nación fue la ya mencionada caída. Sin embargo, en la percepción de los planificadores de PDVSA, eso representaba un logro, era simplemente la disminución de lo que el Gobierno se coge, the government take al cual se referían en el seno de las concesionarias internacionales.

El negocio y los ingresos corporativos crecieron. En los costos se materializaba, según la "meritocracia" de PDVSA "la adecuada remuneración de una empresa capital-intensiva, de una industria moderna y competitiva, del primer mundo, lamentablemente inserta en un país del tercer mundo".

Es oportuno decir que el incremento de esos costos estuvo "condimentado" con la adquisición por PDVSA de 19 refinerías en el exterior, todas suficientemente envejecidas y ampliamente depreciadas como para ser desincorporadas de los activos de sus propietarios originales, las cuales ameritaron inversiones mil millonarias en dólares, a la postre pérdidas netas para la Nación, para ponerlas en condiciones medianas de operación.

La posesión de esas plantas determinó, además de un suministro de crudos nacionales con descuentos respecto a los precios de mercado, el que, a partir de los años 80, un rubro fundamental de los costos consolidados de PDVSA fuera, y sigue siendo hoy, la compra de crudos en el extranjero para alimentar esas refinerías. ${ }^{\mathrm{XX}}$

La debacle de los precios de fines del Siglo XX afectó a todos los países productores de petróleo, exportadores netos o no y, en su momento, determinó un conjunto de movimientos estratégicos, estimulados entre otros por los productores domésticos estadounidenses, quienes movieron sus hilos con el apoyo del Secretario de Energía de ese país para la época, Bill Richardson, para concertar un acuerdo de recorte de la producción en el cual participaron Arabia Saudita, Noruega, México y, a regañadientes, Venezuela.

Ello se logró después de mucho lobby y varias reuniones antes de la aprobación formal por la OPEP, el 23 de marzo de 1999, de un recorte de 388 mil barriles diarios, a partir del cual los precios del crudo marcador norteamericano, que promediaban en ese entonces los 13 dólares el barril, comenzaron a repuntar, y alcanzaron a finales de ese mismo año un promedio de 27 dólares. Algunos analistas consideran que este fue el inicio del superciclo alcista que, mediando el hiato de la crisis del 2008, no se detuvo sino hasta el 2014, cuando alcanzaron a promediar los 110 dólares el barril.

En cuanto a Venezuela, vale la pena destacar que al momento de suscribir "in extremis" los acuerdos de 1999, el Ministro de Energía y Minas y el Presidente de PDVSA, manifestaron la inconformidad "de la industria", anunciando que a mediados del año 2000 PDVSA volvería a producir a plena capacidad. Un anuncio que no tenía ningún sustento, ya que la nueva administración, ya electa en diciembre de 1998, asumió pocos días después, entusiastamente, la política de defensa de los precios. A finales del 2000, se realizó en Caracas la II Cumbre de la OPEP, convocada por el Presidente Chávez para ratificar ese compromiso.

La historia hasta 2005 es harto conocida. Pero el alza de los precios despertó nuevamente el sueño productivista a ultranza y esta vez, como siempre, fundado en la fabulosa Faja del Orinoco, donde yace más de la quinta parte del petróleo del mundo, 


\section{POLÍTICA PETROLERA VENEZOLANA EN TIEMPOS DE CATÁSTROFE}

\section{CARLOS MENDOZA POTTELLÁ}

Como ya referí al inicio, el Servicio Geológico de los Estados Unidos conoce, desde hace décadas, la magnitud de los recursos de hidrocarburos depositados en la ribera norte del Orinoco, los cuales llegaron a ser última esperanza de la civilización automovilístico-petrolera, dada la "crisis energética" de los años 70.

Recientemente, ese Servicio publicó sus estimaciones de "recursos recuperables" en esa Faja, que se fundamentan en la existencia de un "petróleo originalmente en sitio", estimado entre 900 mil millones y 1.400 millones de barriles ${ }^{\text {XXI }}$ :

La tentación de convertir esos "recursos recuperables no descubiertos" en "reservas probadas" ha acompañado a la gerencia de petrolera desde sus inicios consulares y hasta nuestros días:

El referido "megadisparate" de la PDVSA de 1977-83 se fundaba en ese sueño. En 1991 los planificadores de "la apertura" lo registraban en sus estimaciones de "reservas probables" en la Faja del Orinoco por 330 mil millones de barriles.

$\mathrm{Y}$ en 2005, al calor de la recuperación de los precios, se dio inicio al plan "Magna Reserva" para "certificar" las "reservas probadas" que ya se daban por existentes. Uno de los fundamentos de ese plan era la de corregir una supuesta escasa visión de las administraciones del Siglo XX, las cuales estimaron un "escuálido y antipatriótico" factor de recuperación de $4 \%$, el cual resultaba en unas reservas "muy modestas" cercanas a los 30 mil millones de barriles. ${ }^{\text {XXII }}$

Al inicio del plan se le pagó a una empresa, Ryder Scott, para que "certificara" las reservas ya previstas al detalle, con la perforación justificativa de algunos pozos estratigráficos. Dada esa maniobra, más del 90\% de las "reservas" incorporadas entre 2006 y 2016 fueron de la Faja Petrolífera, producto de revisiones y extensiones, muy poco o casi nada por exploración propiamente dicha en nuevas provincias de condensados, medianos y livianos. Se reprocesó información sísmica 2D y 3D pero ello no se tradujo en éxito sustancial alguno en la adición notable de reservas frescas.

La propia PDVSA confiesa inadvertidamente el fiasco de la certificación. Por la módica suma de 557 millones de dólares se perforaron 146 pozos para incorporar, con los datos obtenidos superficialmente y vía revisión de escritorio, 219 mil millones de barriles a las "reservas probadas”. ¡El negocio del milenio! 
POLÍTICA PETROLERA VENEZOLANA EN TIEMPOS DE CATÁSTROFE

CARLOS MENDOZA POTTELLÁ

\begin{tabular}{|c|c|c|c|c|c|c|c|}
\hline & \multicolumn{2}{|c|}{ Resultados de la } & \multirow[b]{3}{*}{ Revisiones } & \multirow[b]{3}{*}{ Producción } & & & \\
\hline & \multicolumn{2}{|c|}{ Exploración } & & & \multicolumn{2}{|c|}{ Reservas } & \multirow{2}{*}{$\begin{array}{c}\text { Relaciòn } \\
\text { R/T }\end{array}$} \\
\hline & Descubrimientos & Extensiones & & & Probadas & Desarrolladas & \\
\hline 1999 & 140 & 98 & 1.622 & 1.117 & 76.852 & & 69 \\
\hline 2000 & 71 & 217 & 1.696 & 1.151 & 77.685 & & 67 \\
\hline 2001 & 112 & 440 & 766 & 1.220 & 77.783 & & 64 \\
\hline 2002 & 2 & 0 & 462 & 1.093 & 77.154 & & 71 \\
\hline 2003 & 274 & 218 & 520 & 1.026 & 77.140 & & 75 \\
\hline 2004 & 319 & 145 & 4.129 & 1.151 & 80.582 & & 70 \\
\hline 2005 & 144 & 35 & 444 & 1.193 & 80.012 & & 67 \\
\hline 2006 & 82 & 31 & 8.383 & 1.184 & 87.324 & & 74 \\
\hline 2007 & 494 & 20 & 12.686 & 1.147 & 99.377 & & 87 \\
\hline 2008 & 8 & 116 & 74.013 & 1.191 & 172.323 & & 145 \\
\hline 2009 & 22 & 0 & 39.925 & 1.097 & 211.173 & 15.054 & 193 \\
\hline 2010 & 200 & 0 & 86.211 & 1.083 & 296.501 & 14.248 & 274 \\
\hline 2011 & 27 & 35 & 2.097 & 1.089 & 297.571 & 13.652 & 273 \\
\hline 2012 & 123 & 0 & 1.105 & 1.064 & 297.753 & 12.975 & 280 \\
\hline 2013 & 162 & 0 & 1.512 & 1.056 & 298.353 & 12.960 & 283 \\
\hline 2014 & 201 & 0 & 2.414 & 1.015 & 299.953 & 12.926 & 296 \\
\hline 2015 & 62 & 0 & 1.864 & 1.001 & 300.878 & 12.931 & 301 \\
\hline 2016 & 42 & 2 & 2.222 & 901 & 302.250 & 12.944 & 335 \\
\hline \multirow[t]{3}{*}{ TOTALES } & 2.485 & 1.357 & 242.071 & 19.779 & & & \\
\hline & \multicolumn{6}{|c|}{ Reservas Probadas 1999 más Descubrimientos y extensiones y menos producción: } & 60.915 \\
\hline & Reservas Probada & as 2016 menos $\mathrm{F}$ & evisiones & & & & 60.179 \\
\hline & & & & & & & \\
\hline
\end{tabular}

En sus tiempos, Juan Pablo Pérez Alfonzo satirizaba a los técnicos del Ministerio de Minas cuando presentaban cifras abultadas de revisiones, les decía que habían afilado los lápices. Ahora, basta con cambiar un porcentaje en una tabla Excel, de 4\% de factor de recobro a $20 \%$.

En esos cálculos, basados en las supuestas posibilidades técnicas de recuperación del petróleo en sitio, se minimiza el pequeño inconveniente de la rentabilidad del negocio. Costos, precios, tasa interna de retorno, valor presente neto, etc., serían problemas que deberá resolver el sector público rentista, minimizando sus apetitos fiscales y dejando al eficiente sector privado optimizar su rentabilidad particular.

Pero, peor aún, cuando se estiman "reservas" para una duración de siglos, se pierde toda significación económica, tanto para la Nación como para cualquier empresa privada, en cualquier escenario previsible de los hidrocarburos como fuentes energéticas.

Reservas para 618 años, las cuales, al doble de la tasa de producción actual se agotarán en un $6,48 \%$ en 100 años $\mathrm{y}$, desde luego, en un $20 \%$ transcurridos seis siglos. XXIV

Lo cierto del caso es que sobre esas endebles bases se reiniciaron en 2005, como dije, las metas expansivas:

El Plan de Negocios 2005-2012, partiendo de la producción de 3,3 millones de barriles diarios en el año inicial, alcanzaría una producción de 5,8 millones en 2012. Contra lo previsto, la producción en ese año fue inferior a la del inicial, 2005. Sin embargo, se siguió insistiendo, y en la nueva oportunidad, a partir de 3,1 millones de bd en 2012 se programó una nueva meta para 2018: 6,8 millones de barriles diarios. Cuatro de esos nuevos millones diarios provendrían de la Faja del Orinoco. XXV 


\section{POLÍTICA PETROLERA VENEZOLANA EN TIEMPOS DE CATÁSTROFE}

\section{CARLOS MENDOZA POTTELLÁ}

Tratando de utilizar el bisturí sin matar al paciente, no me ha quedado otra manera de presentar mis argumentos que exponiendo sin subterfugios la irrealidad de estos planes desde 1978 hasta hoy. El último de esos proyectos conocidos es paradigmático en este sentido. Considérese nada más el exabrupto de las cifras de inversiones que sustentan un crecimiento anual de la producción, también mítico, de 628 mil barriles diarios cada año entre 2014 y 2019:

Un desembolso por inversiones de 302 mil millones de dólares en 5 años, 60 mil millones de dólares anuales, fuera de todas las posibilidades de una industria y un país cuyos excedentes netos mermaban cada año, y apenas superaron los 6.000 millones de dólares en el último año del cual se tienen datos, 2016.

Es realmente descomunal la brecha entre la realidad y las metas planteadas por esta planificación de ensueños tipo Disneyworld. Planificación que ha resultado en una auténtica tragedia nacional, irrecuperable en cualquier plazo previsible, porque los daños permanentes a nuestra Nación y a su futuro, producto de "inversiones" completamente irrecuperables, fueron y serán incuantificables.

¿Cuánto del desembolso planificado se "ejecutó" para obtener este resultado catastrófico? ¿En qué cuentas de cuáles corporaciones e individualidades se encuentran hoy esos despilfarrados recursos públicos?

Más allá de ese cálculo crematístico, se trata de la afectación perdurable de los derechos colectivos, de lo que los juristas denominan "el interés difuso". Y es precisamente ese carácter difuso el que hace que los individuos no perciban su responsabilidad en la protección de esos derechos y ese patrimonio porque, "lo que es de todos no es de nadie".

Dejando atrás los lamentos, debemos volver al corazón del debate que las motivó inicialmente: el enfrentamiento entre los escenarios "productores" y "rentistas".

Cuando observamos gráficamente el curso creciente de la participación de los costos en los ingresos totales y la consecuente minimización de la participación fiscal, incluidos los "aportes sociales", podemos inferir hacia dónde fue la renta extraordinaria generada en la industria petrolera en las pasadas décadas: 
PDVSA: ESTADO CONSOLIDADO DE GANANCIAS Y PÉRDIDAS 1997 - 2016
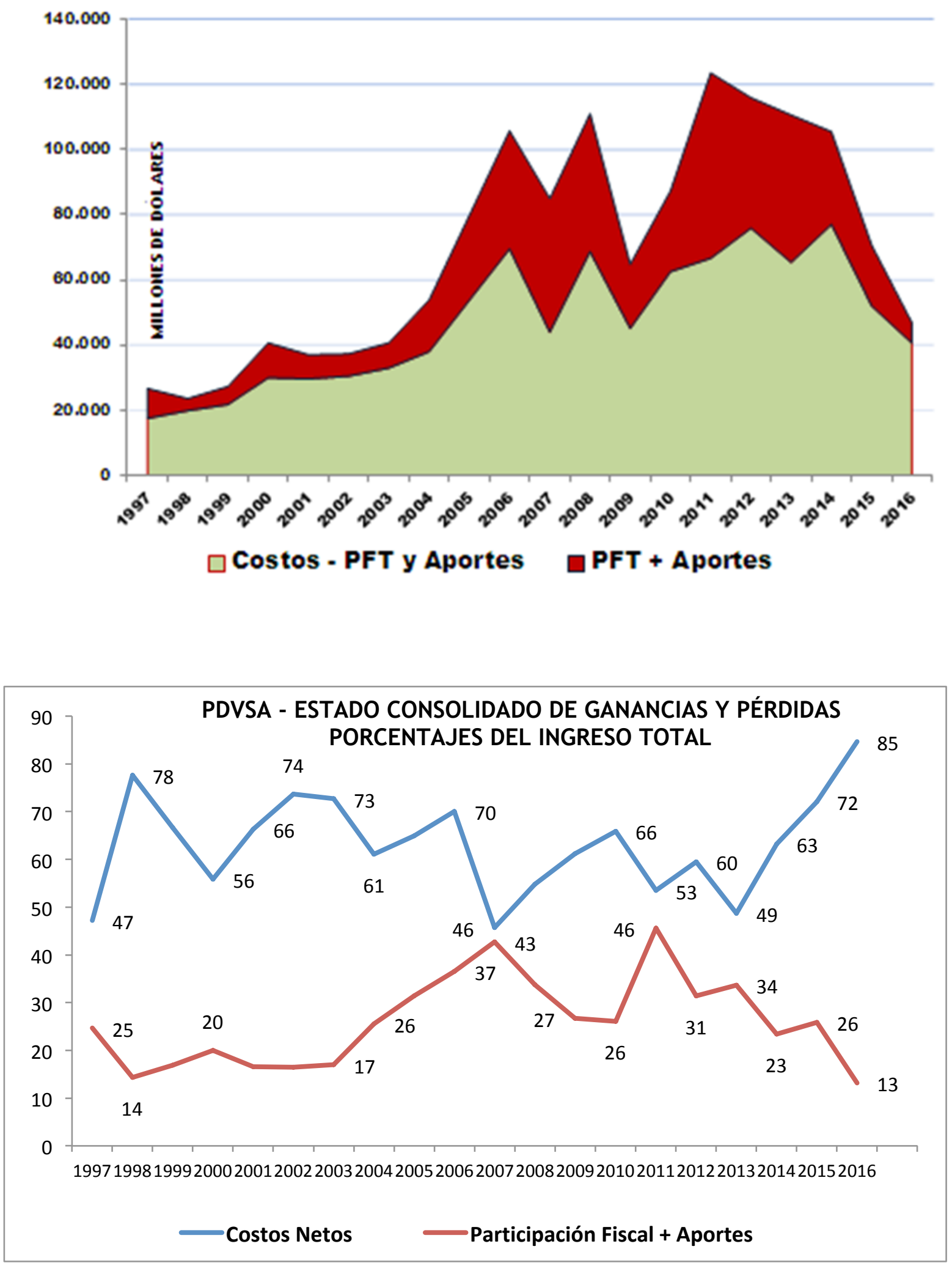

Es pertinente traer estas cifras al debate, porque una de las más socorridas explicaciones de la debacle de la industria petrolera venezolana se centra en demonizar 


\section{POLÍTICA PETROLERA VENEZOLANA EN TIEMPOS DE CATÁSTROFE}

\section{CARLOS MENDOZA POTTELLÁ}

la participación nacional en los proventos de la misma y en una presentación falaz de las cifras de resultados, que justifica la reiterada solicitud, por parte de sectores privados e ideológicamente privatistas, de disminución de la "excesiva carga fiscal" que pesa sobre la industria petrolera.

Podemos ahora preguntar ¿en qué parte de las cifras que generan los dos gráficos anteriores se esconde esa excesiva carga? ¿no son esos costos, cuya participación en el reparto ya pasa del 86 por ciento del ingreso total, el reflejo parcial de la creciente tajada que toman para sí las empresas privadas nacionales e internacionales que participan en el negocio, más allá del pago de los suministros que aportan, la remuneración de sus inversiones y de los servicios que realizan?

Está completamente claro aquí que esas exigencias de reducción de la carga fiscal no son otra cosa más que la perenne pugna por una mayor participación privada en la apropiación del excedente que se genera en la industria petrolera, la maximización de sus beneficios. En esa pugna, las corporaciones internacionales fueron exitosas durante los últimos cien años. Basta solo con revisar el "ranking" histórico de las mayores empresas industriales del mundo y encontrar allí los puestos que han ocupado y ocupan las corporaciones petroleras y de servicios conexos, tales como Exxon-Mobil, Shell, BP, Conoco-Phillips, Chevron, Total, Schlumberger, Halliburton, etc.

De cómo lo hicieron, y como fue la participación de sus Estados metropolitanos en la implantación de su poder monopólico global, tratan muchos volúmenes escritos desde los años 30 del Siglo pasado. No los vamos a citar aquí, pero vale la pena recordar que Venezuela fue y sigue siendo un principalísimo escenario de ese proceso, donde la geopolítica de las grandes potencias juega un papel preponderante.

Esta es una acotación pertinente, también, para refrescar la memoria de quienes plantean el asunto, con aparente ingenuidad, como un simple debate entre el atraso y la modernidad, entre perversos rentistas y estatistas enfrentados a los promotores de la iniciativa privada creadora de valor.

Contemporáneamente, esa confrontación se agudiza por la circunstancia de que los excedentes actuales de esa explotación en el país ya no pueden ser catalogados como "renta", dado el margen cada día más estrecho entre costos y precios. A unos niveles tales que hacen inviables, en el corto y mediano plazo, la expansión de operaciones extractivas en los inmensos yacimientos de crudos extrapesados que impregnan el subsuelo venezolano. 
POLÍTICA PETROLERA VENEZOLANA EN TIEMPOS DE CATÁSTROFE

CARLOS MENDOZA POTTELLÁ

\section{Precio mínimo del barril de WTI para justificar inversiones en nuevos proyectos}

\begin{tabular}{|c|c|}
\hline Localización & USD/B \\
\hline Medio Oriente & 20 \\
\hline China & 28 \\
\hline Libia & 42 \\
\hline México & 55 \\
\hline Brasil & 61 \\
\hline Aguas Profundas Golfo de & 65 \\
México/EEUU & \\
\hline Angola & 71 \\
\hline Nigeria & 78 \\
\hline Arenas Bituminosas Canadá & 87 \\
\hline Crudo extrapesado de Venezuela & 114 \\
\hline \multicolumn{2}{|c|}{ Fuente: CERA } \\
\hline
\end{tabular}

No es necesario citar los múltiples escenarios que presentan un panorama de estancamiento de la demanda petrolera y precios deprimidos en las próximas décadas, para estimar la inviabilidad de cualquier nuevo desarrollo en la Faja del Orinoco.

Sin colocarme gríngolas para eludir el trasfondo ideológico de esta discusión, ni, como es evidente en este trabajo, voy a esconder que mantengo una posición enfrentada a las visiones liberales sobre la administración y disposición de un patrimonio nacional, vale decir social, colectivo y de incumbencia transgeneracional por su magnitud y perdurabilidad.

Se trata de combatir las propuestas que involucran una pulverización del Estado Nación venezolano, en medio de las pugnas de los más grandes Estados mundiales por someter a los más débiles a sus particulares designios económicos y políticos. Esa es la principal motivación de los trabajos que he sometido al escrutinio público en los últimos años. XXVII 


\section{CARLOS MENDOZA POTTELLÁ}

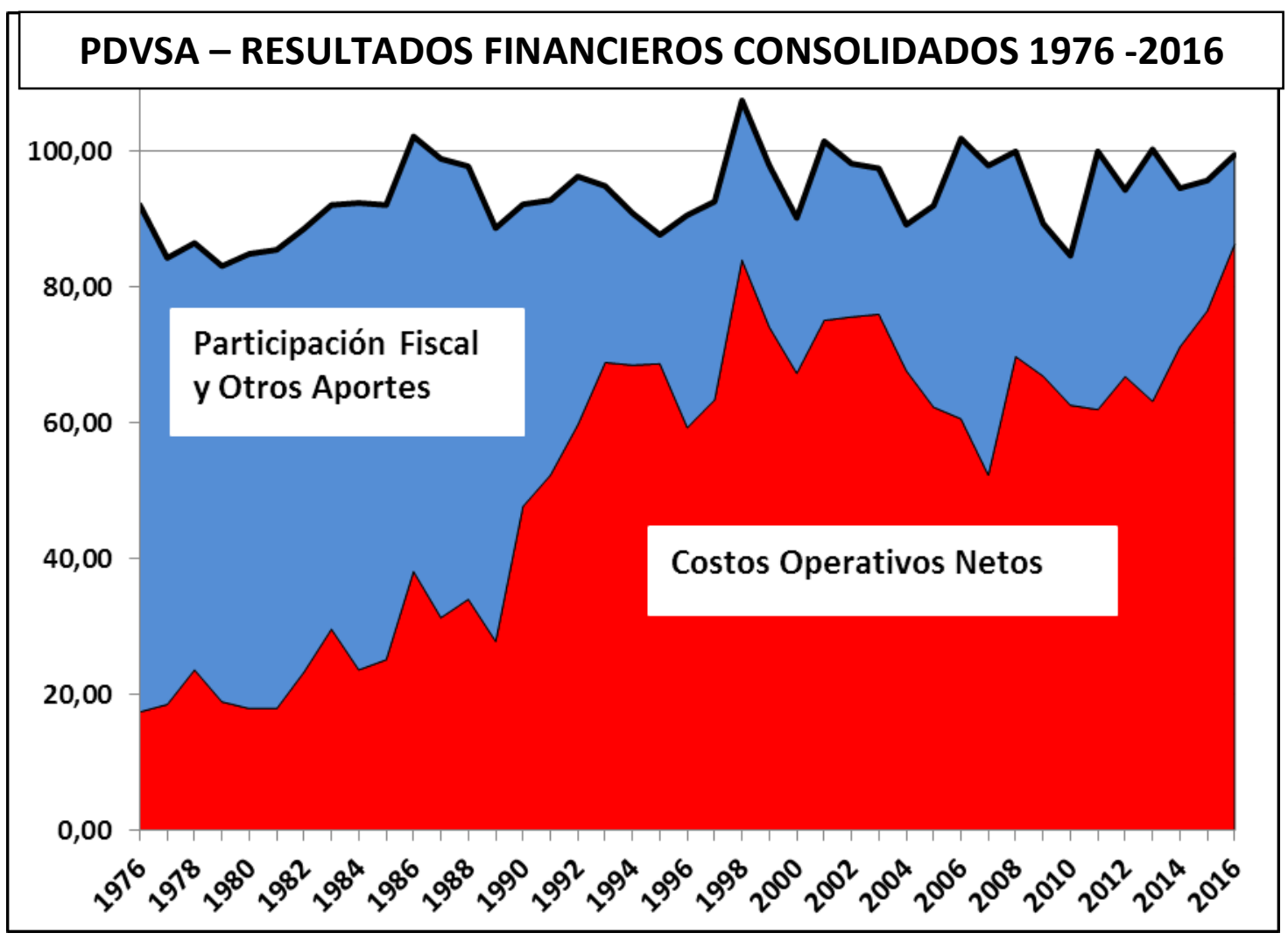

CMP 21/10/19

\section{Notas}

I Economista (Magíster Scientiarum en Economía y Administración de los Hidrocarburos). Profesor Asociado de la Universidad Central de Venezuela (Jubilado). Asesor de la Presidencia del Banco Central de Venezuela. Director de la Revista BCV. Coordinador del Equipo de Estudios Petroleros del BCV

II Juan Pablo Pérez Alfonzo, Revista "Semana", Vol. XXI, No 235, Caracas 1978. Reproducido en Petróleo y Ecodesarrollo en Venezuela, Dorothea Mezger (Compiladora), ILDIS, Caracas 1981 y en el Suplemento de la Revista BCV -- 1, Enero-Junio 2008, "Profecías Cumplidas", Banco Central de Venezuela, Caracas 2008.

III "Maldición de los Recursos" y "Enfermedad Holandesa", fueron los términos utilizados por los economistas que "descubrieron", en los años 70, el fenómeno padecido por Venezuela desde 1920.

IV Ministerio de Minas e Hidrocarburos, Petróleo y Otros Datos Estadísticos Caracas 1959-2007 PDVSA, Inforne General de Actividades, 2008-2016.

${ }^{\mathrm{V}}$ Richard F.Meyer and Emil D. Attanasi, US Geological Survey, Heavy Oil and Natural BitumenStrategic Petroleum Resources. . http://pubs.usgs.gov/fs/fs070-03/fs070-03.html

${ }^{\mathrm{VI}}$ https://petroleovenezolano.blogspot.com/2019/02/recursos-reservas-faja-y-lutitas.html\#.XadRo-ZKjIU

VII . https://elpais.com/diario/1979/07/17/economia/301010411 850215.html

VIII https://www.foreignaffairs.com/articles/middle-east/1973-04-01/oil-crisis-time-wolf-here

${ }^{\mathrm{IX}}$ Ley Sobre Bienes Afectos a Reversión. Congreso de la República de Venezuela, Gaceta Oficial $\mathrm{n}^{\circ}$ 59.577 06/08/71, Editorial La Torre, Caracas 1971.

${ }^{\mathrm{X}}$ Rodríguez G., Irene, Yánez, Francisco, Cronología Ideológica de la Nacionalización Petrolera en Venezuela, Ed. FCES-UCV, Caracas, 1977, págs. 115, 138,145,

${ }^{\mathrm{XI}}$ Mendoza P., Carlos De las Concesiones a los Contratos, visión retrospectiva de la política petrolera venezolana, Tesis de Maestría en Economía y Administración de Hidrocarburos, FACES-UCV, Caracas 1985 Publicado como Análisis crítico de la política petrolera venezolana hasta 1985 Editorial Académica Española, LAP Lambert Academic Publishing Gmbh, Saarbrüken, Alemania, 2011. 


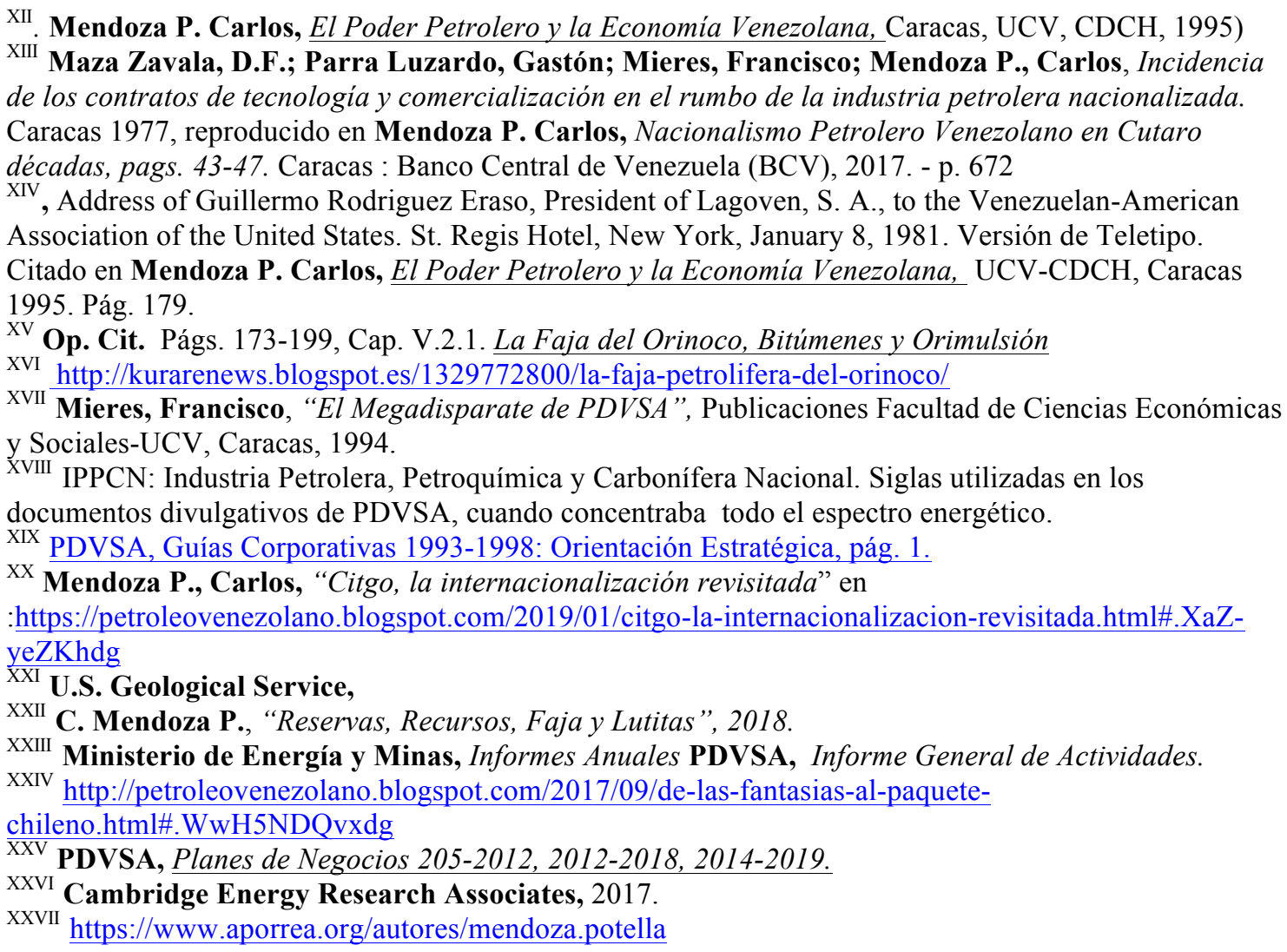

\section{BIBLIOGRAFÍA:}

AKINS, James. The Oil Crisis, This Time de Wolf is Here, Foreign Affairs, Abril 1973. https://www.foreignaffairs.com/articles/middle-east/1973-04-01/oil-crisis-timewolf-here

Congreso de la República de Venezuela. Ley Sobre Bienes Afectos a Reversión., Gaceta Oficial N ${ }^{\circ} 59.577$ 06/08/71, Editorial La Torre, Caracas 1971.

ESPINASA, Ramón. Las Estrategias Petroleras después de la Nacionalización 1976 - 2006. XIII Congreso de Actualidad Económica UCAB Caracas Mayo 2006

GONZALES C, Diego. Propuestas para Venezuela; Cómo rescatar a la industria petrolera nacional. CEDICE Libertad, Caracas 2016.

LÓPEZ, Leopoldo. Baquero Gustavo.Venezuela Energética. Propuesta para el bienestar y el progreso de los venezolanos. CEDICE Libertad Caracas 2019.

MAZA ZAVALA, D.F., Parra Luzardo, Gastón, Mieres, Francisco, Mendoza P., Carlos. Incidencia de los contratos de tecnología y comercialización en el rumbo de la industria petrolera nacionalizada. Caracas 1977, reproducido en Mendoza P. Carlos, Nacionalismo Petrolero Venezolano en Cuatro décadas, pags. 43-47. Banco 


\begin{tabular}{llll}
\hline Central de & Venezuela & Caracas
\end{tabular}

2017. http://publicaciones.extra.bcv.org.ve/colecciones.html.

MENDOZA POTTELlÁ, Carlos. De las Concesiones a los Contratos, visión retrospectiva de la política petrolera venezolana, Caracas 1985. / Editorial El Perro y la Rana, Caracas 2012. http://petroleovenezolano.blogspot.com/2009/12/de-lasconcesiones-los-contratos-vision.html

----- Análisis crítico de la política petrolera venezolana hasta 1985 Editorial Académica Española, LAP Lambert Academic Publishing Gmbh, Saarbrüken, Alemania, 2011/

----- El Poder Petrolero y la Economía Venezolana, Universidad Central de Venezuela, CDCH, Caracas, 1995).

----- Crítica Petrolera Contemporánea, Universidad Central de Venezuela, FACES, Caracas 1999. https://petroleovenezolano.blogspot.com/2009/12/critica-petroleracontemporanea_24.html\#.XhYVm9JKjIU

----- Nacionalismo Petrolero Venezolano en Cuatro décadas, Banco Central de Venezuela (BCV), Caracas 2017.

---- https://publicaciones.extra.bcv.org.ve/colecciones.html

----- https://petroleovenezolano.blogspot.com

---- https://www.aporrea.org/autores/mendoza.potella

MEYER, Richard F. and Attanasi Emil D. Heavy Oil and Natural Bitumen-Strategic Petroleum Resources. US Geological Survey , http://pubs.usgs.gov/fs/fs07003/fs070-03.html

MIERES, Francisco. El Megadisparate de PDVSA, Publicaciones Facultad de Ciencias Económicas y Sociales-UCV, Caracas, 1994.

Ministerio de Minas e Hidrocarburos - Ministerio de Energía y Petróleo, ---- Petróleo y Otros Datos Estadísticos , Caracas 1959-2008

OLIVEROS LUIS, Coordinador. La industria petrolera en la era chavista, crónica de un fracaso, Instituto de Estudios Parlamentarios Fermín Toro, Universidad Católica Andrés Bello, Caracas 2019.

PARRA LUZARDO, Gastón. De la Nacionalización a la apertura petrolera, Banco Central de Venezuela, Colección Venezuela y su petróleo Caracas, 2008.

Petróleos de Venezuela S. A., PDVSA, ---- Guías Corporativas 1993-1998: Orientación Estratégica, (Bernard Mommer y Ramón Espinasa, Planificadores Mayores)

---- Informe General de Actividades, 2008-2016. 
---- Planes de Negocios 205-2012, 2012-2018, 2014-2019.

PÉREZ ALFONZO, Juan Pablo. Venezuela se acerca a la debacle, Entrevista en Revista "Semana", Vol. XXI, N ${ }^{\circ}$ 235, Caracas 1978. Reproducido en Petróleo y Ecodesarrollo en Venezuela, Dorothea Mezger (Compiladora), ILDIS, Caracas 1981 y en el Suplemento de la Revista BCV 1, Enero-Junio 2008, “Profecías Cumplidas”, Banco Central de Venezuela, Caracas 2008.

---- Hundiéndonos en el excremento del diablo, Colección Venezuela y su petróleo , Banco Central de Venezuela, Caracas 2011.

RODRIGUÉZ G., Irene, Yánez, Francisco. Cronología Ideológica de la Nacionalización Petrolera en Venezuela, Ed. FCES-UCV, Caracas, 1977,

US Geological Survey, ---- An Estimate of Recoverable Heavy Resources of the Orinoco Oil Belt, Venezuela. World Petroleum Resources Project, Fact Sheet 2009 3028, October 2009.

VALECILlOS, Héctor y BELlO, Omar La Economía Contemporánea de Venezuela. Alberto Adriani , La crisis, los cambios y nosotros, Tomo I, págs. 21-42., Banco Central de Venezuela, Colección Cincuentenaria, Caracas, 1990. 